\title{
Parathyroid Gland Atypical Adenoma
}

National Cancer Institute

\section{Source}

National Cancer Institute. Parathyroid Gland Atypical Adenoma. NCI Thesaurus. Code C48285.

A parathyroid gland tumor that shares certain morphologic characteristics with parathyroid gland carcinomas (e.g. broad fibrous bands formation with or without hemosiderin deposition, presence of mitotic figures) but lacks unequivocal evidence of capsular or vascular invasion. It is considered a tumor of uncertain malignant potential (adapted from WHO, IARC Press, Lyon, 2004). 